
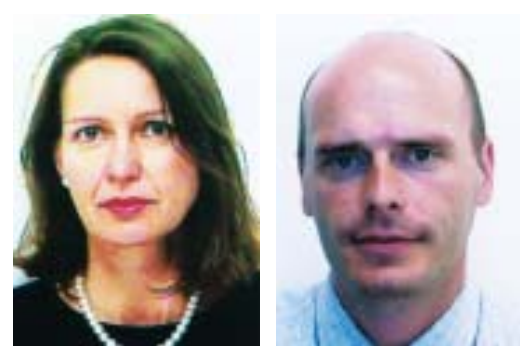

Marcella Rietschel Markus M. Nöthen

\section{Genetik bipolar affektiver Störungen}

\author{
Marcella Rietschel ${ }^{1,2}$ und Markus M. Nöthen ${ }^{3}$ \\ ${ }^{1}$ Klinik und Poliklinik für Psychiatrie und Psychotherapie, Universität Bonn \\ 2Zentralinstitut für Seelische Gesundheit, Mannheim \\ ${ }^{3}$ Department of Medical Genetics, University of Antwerp
}

psychoneuro 2003; 29 (4): 171-174
B ipolar affektive Störungen, auch bekannt unter dem Begriff „manisch depressive“ Störungen, sind Gemütserkrankungen, die sich durch einen Wechsel der Gemütslage zwischen zwei (bi) Polen auszeichnen: zwischen der Manie und der Depression. Dieser Wechsel kann sich sehr rasch, innerhalb von Stunden bis Tagen, vollziehen, es können aber auch lange Phasen von einigen Monaten bis Jahren zwischen den Phasen liegen.

Die Stimmung in der Depression ist niedergedrückt, freudlos, traurig. Antriebsarmut, Denkverlangsamung, Schuldgefühle und Lebensüberdruss begleitet von Schlaf- und Appetitstörungen sind weitere Charakteristika. Die Manie hingegen ist gekennzeichnet durch eine extrem gehobene, teilweise auch gereizte Stimmung mit Antriebssteigerung, vermehrtem Gedanken- und Sprachfluss, Selbstüberschätzung und expansivem Verhalten.

In Abhängigkeit vom Schweregrad der Manie unterscheidet das moderne Diagnosesystem DSM-IV (1) zwischen einer Bipolar-I-Störung mit einer klinisch stark ausgeprägten manischen Symptomatik, die auch

Bipolar affektive Störungen, die durch einen Wechsel von depressiven und manischen Krankheitsepisoden gekennzeichnet sind, zählen zu den komplex bedingten Erkrankungen. Das heißt, sowohl Umwelteinflüsse als auch Gene beeinflussen die Krankheitsdisposition und -entwicklung. Die Suche nach den Vulnerabilitätsgenen konzentriert sich zurzeit auf chromosomale Regionen, für die in unabhängigen Kopplungsuntersuchungen Evidenz für das Vorhandensein solcher Gene gefunden wurde. Die Kenntnis dieser Gene wird zu einem besseren Verständnis der Pathophysiologie der Erkrankung führen und die Voraussetzungen zur Untersuchung von Gen-Umwelt-Interaktionen schaffen. Man hofft, dass die dabei erzielten Erkenntnisse die Grundlage zur Entwicklung neuer kausaler und individueller Therapien legen werden.

wahnhafte Symptome beinhalten kann, und einer Bipolar-II-Störung mit schwächer ausgeprägten, sog. hypomanen Symptomen. Treten keine manischen, sondern nur depressive Phasen auf, wird die Diagnose einer unipolaren Depression gestellt. Auch wenn die depressiven Episoden bei bipolaren und unipolaren Störungen klinisch nicht zu unterscheiden sind, deuten der Verlauf, die Behandlung und die formalgenetischen Befunde darauf hin, dass es sinnvoll ist, bipolare und unipolare Störungen getrennt zu betrachten.

Bipolare affektive Störungen sind sowohl schwere als auch häufige Erkrankungen. Die Lebenszeitprävalenz der Bipolar-I-Störung beträgt $0,5-1 \%$, wobei sich im Laufe des Lebens ca. 10\%-15\% der Betroffenen suizidieren. Trotz wirksamer Medikamente ist die Therapie immer noch unzureichend, da die Ursachen der Erkrankung trotz intensivster Forschung noch nicht bekannt sind.

Dass genetische Faktoren ganz erheblich zur Entstehung der bipolar affektiven Störungen beitragen, wird durch eine große Anzahl formalgenetischer Untersuchungen nahe gelegt. Deren Ergebnisse wollen wir im Folgenden kurz zusammenfassen und auch auf derzeit laufende Untersuchungen zur Identifikation der genetischen Faktoren auf molekularer Ebene hinweisen.

Als formalgenetische Untersuchungen werden die klassischen Fa- 
milien-, Zwillings- und Adoptionsstudien bezeichnet. In der Bemühung den genetischen Beitrag einer Erkrankung festzustellen, sind diese Untersuchungen komplementär.

\section{Formalgenetische \\ Untersuchungen \\ Familienstudien}

Bei der Mehrzahl der früheren Familienstudien wurde nicht zwischen bipolar und unipolar affektiven Störungen unterschieden. Die Ergebnisse aller neueren Familienstudien, die mit standardisierten Methoden durchgeführt wurden und das Konzept der bipolaren Störungen berücksichtigten, weisen darauf hin, dass erstgradig Verwandte von Patienten mit einer Bipolar-I-Störung ein erhöhtes Risiko haben, ebenfalls eine bipolare Störung zu entwickeln. Im Mittel liegt das relative Risiko eines erstgradig Verwandten hierfür bei ca. 7 . Das relative Risiko ist definiert als das Verhältnis des Risikos eines erstgradig Verwandten eines Erkrankten zu dem Risiko eines erstgradig Verwandten eines Kontrollprobanden.

Diese Studien zeigen ebenfalls übereinstimmend, dass nicht nur das Risiko für bipolare, sondern auch für unipolare Störungen in diesen Familien erhöht ist (Tab. 1), wobei sich das Risiko für unipolare Störungen ungefähr verdoppelt. Allerdings ist es im Einzelfall nicht möglich zu entscheiden, ob die jeweilige unipolare Störung auf dem Boden einer familiären genetischen Belastung für eine bipolare Störung entstand, da unipolare Störungen mit einer Lebenszeitprävalenz von $5-10 \%$ insgesamt sehr häufig in der Allgemeinbevölkerung auftreten. Es gibt jedoch Schätzungen, die davon ausgehen, dass ca. 70\% dieser familiären
Fälle auf eine gemeinsame genetische Belastung zurückzuführen sind (3). In den Familien bipolar Erkrankter kommen unipolare Depressionen bei Frauen etwa doppelt so häufig vor wie bei Männern, während das Risiko für bipolare Störungen für beide Geschlechter gleich hoch ist dieses Phänomen ist auch in der Allgemeinbevölkerung zu beobachten.

Auch Bipolar-II-Störungen treten in Familien von Betroffenen häufiger auf als in der Allgemeinbevölkerung $(8,11,20)$. Es ist bislang nicht klar, ob Bipolar-II-Störungen lediglich eine phänotypische Variante der BipolarI-Störungen darstellen oder ob sie, ähnlich wie unipolare Depressionen, auch ohne diesen genetischen Hintergrund auftreten $(8,11,13,15,20)$.

Kontrovers ist auch, ob das Risiko eines erstgradig Verwandten sich in Abhängigkeit davon unterscheidet, ob er ein Geschwister, ein Kind oder ein Elternteil des Betroffenen ist $(8,9)$.

Während das Risiko für die Entwicklung einer bipolaren Störung unabhängig vom Geschlecht des Betroffenen oder des Angehörigen ist, steigt es, mit der Anzahl der Betroffenen in der Familie und bei einem frühen Beginn der Erkrankung des betroffenen Familienangehörigen.

Familienangehörige zweiten Grades haben ein weitaus geringeres Risiko, welches nahe bei dem der Allgemeinbevölkerung liegt.

Segregationsuntersuchungen zeigen, dass das in den Familien beobachtete Vererbungsmuster nicht kompatibel mit einer monogenen Übertragung ist.

Zudem ist die Beobachtung einer familiären Häufung einer Erkrankung an sich noch kein Nachweis dafür, dass diese genetisch bedingt ist. Eine andere mögliche Erklärung hierfür könnte beispielsweise sein, dass früh erlernte Verhaltensmuster bzw. die häusliche Belastung zur Ausbildung vergleichbarer Symptome bei Familienangehörigen führen. Aufschluss über einen tatsächlichen genetischen Beitrag lieferten hauptsächlich die Ergebnisse von Zwillingsstudien.

\section{Zwillingsuntersuchungen}

Während eineiige Zwillinge annähernd $100 \%$ ihres genetischen Materials gemein haben, teilen zweieiige Zwillinge - so wie normale Geschwister - im Schnitt 50\% ihres Erbgutes. Bei einer genetisch (mit)bedingten Erkrankung müssen die Konkordanzraten eineiiger Zwillinge höher sein als die zweieiiger. Die Konkordanzraten betragen für eineiige Zwillinge im Mittel 50\% für bipolare Störungen und $80 \%$, wenn die Diagnose des Ko-Zwillings bipolare bzw. affektive Störung lautet (Tab. 2). Das Ausmaß der Heritabilität (d.h. die durch genetische Faktoren erklärbare Varianz) beträgt bei bipolar affektiven Störungen 6080\%; bei unipolaren Depressionen zeigen neuere Untersuchungen ähnlich hohe Werte (16).

\section{Adoptionsuntersuchungen}

Bislang wurden lediglich zwei Adoptionsstudien spezifisch in Hinblick auf bipolare Störungen durchgeführt (19, 27). In der Studie von Mendlewicz und Rainer (19) wurden die biologischen und die Adoptiveltern gesunder $(n=22)$ und bipolar erkrankter $(\mathrm{n}=29)$ Adoptivkinder und als Kontrollgruppe Eltern von bipolar erkrankten $(n=31)$ und psychisch gesunden ( $n=20)$, aber an Kinderlähmung erkrankten Kindern untersucht. Es wurden deshalb Kinder mit Kinderlähmung gewählt, um einen

\section{Tab. 1 Lebenszeitrisiko für affektive Störungen für erstgradig Verwandte von bipolar affektiv Erkrankten und für die Allgemeinbevölkerung}

Verwandtschaftsgrad zum bipolar
affektiv Erkrankten

Eineiiger Ko-Zwilling

Erstgradig Verwandter

Allgemeinbevölkerung
Risiko für bipolar affektive Störung
(Zusätzliches) Risiko für unipolar depressive Störung

$$
\begin{array}{r}
15-25 \% \\
10-20 \% \\
5-10 \%
\end{array}
$$

nach Craddock und Jones 1999(17) 
möglichen Effekt kontrollieren zu können, der durch die Betreuung eines kranken Kindes entstehen könnte. Auch wenn die Anzahl der Untersuchten relativ gering war, weisen die Ergebnisse auf einen genetischen Beitrag hin: Von den biologischen Eltern der 29 erkrankten Adoptivkinder hatten 18 (31\%) eine affektive (bipolare, schizoaffektive oder unipolare Störung) Erkrankung, von den Adoptiveltern waren es lediglich 7 (12\%). Von den Eltern der 31 nicht weg-adoptierten erkrankten Kinder waren 16 (25\%) erkrankt. Das Risiko der Eltern psychisch gesunder Kinder unterscheidet sich nicht von dem der Adoptiveltern gesunder und kranker Kinder.

In der aufwändigen Adoptionsuntersuchung von Wender und Kollegen (27) wurden Eltern von affektiv erkrankten $(\mathrm{n}=31)$ und gesunden $(\mathrm{n}=71)$ Kindern, die nach Alter, Geschlecht, Adoptionszeitpunkt und sozialem Status parallelisiert worden waren, untersucht. Die Ergebnisse weisen zwar deutlich auf die Beteiligung genetischer Faktoren hin, da aber von den erkrankten Kindern nur zehn eine bipolare Störung aufwiesen, können keine spezifischen Aussagen für die bipolaren Störungen gemacht werden.

\section{Molekulargenetische Untersuchungen} Kopplungsuntersuchungen

Bereits zu einer Zeit als noch nicht die Möglichkeit bestand, mit molekulargenetischen Markern Kopplungsuntersuchungen durchzuführen, berichtete die Arbeitsgruppe von Mendlewicz Kopplung von bipolaren Störungen mit dem Auftreten von Rot-Grün-Blindheit bzw. mit Glukose-6-PhosphatdehydrogenaseMangel in entsprechenden Familien (18). Diese Befunde, die noch auf dem Konzept der monogenen Vererbung von Erkrankungen basierten, konnten im weiteren Verlauf, als zunehmend mehr molekulargenetische Marker zur Verfügung standen, nicht eindeutig bestätigt werden (17). Ähnlich verhielt es sich mit den ersten positiven Kopplungsbefunden, die mittels einiger weniger molekulargenetischer Marker in der „Old Order Amish Familie“ berichtet

\begin{tabular}{lllr} 
Tab. 2 & $\begin{array}{l}\text { Konkordanzraten eineiiger und zweieiiger Zwillinge für } \\
\text { unipolare (UP) und bipolar affektive (BP) Störungen } \\
(\mathbf{2 , 4 , 1 4 )}\end{array}$ \\
\multicolumn{5}{c}{ Index-Zwilling } & $\begin{array}{l}\text { Ko-Zwilling } \\
\text { UP }\end{array}$ & BP \\
\hline Eineiige Zwillinge & UP & $35 \%-42 \%$ & $0 \%-9 \%$ \\
Zweieige Zwillinge & BP & $18 \%-20 \%$ & $50 \%-61 \%$ \\
& UP & $18 \%-20 \%$ & $2 \%-6 \%$ \\
& BP & $5 \%-11 \%$ & $5 \%-8 \%$
\end{tabular}

wurden. Auch diese Befunde $(7,12)$ mussten später teilweise erheblich eingeschränkt werden.

Zum heutigen Zeitpunkt liegen die Ergebnisse von 18 abgeschlossenen genomweiten Kopplungsanalysen vor. Wie für komplexe Erkrankungen $\mathrm{zu}$ erwarten, finden die meisten Autoren in ihren Untersuchungen jeweils mehrere chromosomale Regionen, die gekoppelt sind. Keine dieser Regionen zeigte jedoch einen über alle Analysen hinweg konsistent positiven Befund. Die Regionen, die in einer kürzlich durchgeführten Metaanalyse die höchsten Signifikanzwerte zeigen, liegen auf dem kurzen Arm des Chromosoms 9, dem langen Arm der Chromosomen 10 und 14 und auf dem kurzen und langen Arm des Chromosoms 18 (24).

Die scheinbare Inkonsistenz der Befunde, so wie die Vielzahl von positiven Kopplungsbefunden, lässt sich dadurch erklären, dass mehrere Gene mit unterschiedlicher Stärke zur Entstehung der Krankheit beitragen. Zudem kann davon ausgegangen werden, dass die in den verschiedenen Studien untersuchten Patientenkollektive sowohl in Hinblick auf ihre Erkrankung als auch in Hinblick auf ihre Ethnizität heterogen waren. Somit kann nicht ausgeschlossen werden, dass auch solche Regionen Dispositionsgene enthalten, die niedrigere Signifikanzwerte aufwiesen oder für die nur in einer Studie wiederholt Hinweise auf Kopplung gefunden wurden. Sehr Erfolg versprechend sind natürlich die Regionen, die durch unabhängige Arbeitsgruppen gefunden wurden.

\section{Assoziationsuntersuchungen}

Mit dem so genannten „Kandidatengenansatz" wurden in der Ver- gangenheit eine Vielzahl von Assoziationsuntersuchungen mit so genannten Kandidatengenen (Gene, die auf Grund der pathophysiologischen Relevanz ihrer Genprodukte eine kausale Rolle bei der Entstehung der Erkrankung vermuten lassen, wie z.B. Gene, die für Serotoninrezeptoren kodieren) durchgeführt.

Auch wenn keine der bei diesen Untersuchungen berichteten Assoziationsbefunde überzeugend repliziert werden konnte, sprechen einige Studien für einen möglichen Beitrag zur Entstehung der Erkrankungen durch Varianten in den Genen für den Serotonintransporter und die Catechol-O-Methyltransferase (COMT) (Überblick bei 6 und 21).

Zukünftig werden Kandidatengene nicht mehr (nur) in Hinblick auf ihre mögliche pathophysiologische Bedeutung ausgewählt werden, sondern auf Grund ihrer chromosomalen Lokalisation in Regionen, die positive Kopplungsergebnisse aufweisen. Erste Befunde weisen darauf hin, dass mit dieser Strategie der lange erwartete Durchbruch auf der Suche nach Dispositionsgenen gelingt $(5,10,22,23,25,26)$.

\section{Summary}

Bipolar affective disorder is characterised by a pathological change of mood ranging from depressive to manic episodes. The aetiology is complex, with genetic as well as environmental factors contributing to the disposition and course of the disease. The intensive search for vulnerability genes concentrates on those chromosomal regions for which independent linkage studies have repeatedly provided evidence to host those genes. Knowledge of these genes will provide 


\section{Schwerpunkt}

a better understanding of the disorder's pathophysiology and will allow to investigate gene-environment interactions. It is hoped that these developments will lead to new rational and individualised therapies.

\section{Key Words:}

bipolar disorder - manic-depression

- vulnerability genes - family study twin study - association - linkage risk factor

\section{Literatur}

1. American Psychiatric Association (APA) Diagnostic and Statistical Manual of Mental Disorders, Fourth Edition. Washington D.C. American Pychiatric Association, 1994

2. Bertelsen A, Harvald B et al. A Danish twin study of manic-depressive disorders. Br. J Psychiatry 1977; 130: 330-351

3. Blacker D und Tsuang TMT. Unipolar relatives in bipolar pedigrees: are they bipolar. Psychiatr Genet 1993; 3: 5-16

4. Cardno AG, Marshall EJ et al. Heritability estimates for psychotic disorders: the Maudsley twin psychosis series. Arch Gen Psychiatry 1999; 56: 162-168

5. Chumakov I, Blumenfeld $\mathrm{M}$ et al. Genetic and physiological data implicating the new human gene $G 72$ and the gene for $D$ amino acid oxidase in schizophrenia. Proc Natl Acad Sci U S A 2002; 99: 3675-3680

6. Craddock N und Jones I. Genetics of bipolar disorder. J Med Genet 1999; 36 : 585-594

7. Egeland JA, Gerhard D et al. Bipolar affective disorders linked to DNA markers on chromosome 11. Nature 1987; 325: 783-87 8. Gershon ES, Hamovit J et al. A family study of schizoaffective, bipolar II, unipolar and normal control probands. Arch Gen Psychiatry 1982; 39: 1157-1167

9. Gershon ES, Mark A et al. Transmitted factors in the morbid risk of affective disorders: a controlled study. I Psychiatr Res 1975; 12: 283-299

10. Hattori E, Liu C et al. Polymorphisms of LG72 (on 13q33) associated with bipolar disorder by TDT and partition of linkage evidence. Am J Hum Genet 2002; 71: 194

11. Heun R and Maier W. The distinction of bipolar II disorder from bipolar and recurrent unipolar depression: results of a controlled family study. Acta Psychiatr Scand 1993; 87: 279-284

12. Kelsoe JR, Ginn El, et al. Reevaluation of the linkage relationship between chromosome $11 \mathrm{p}$ loci and the gene for bipolar affective disorder in the Old Order Amish. Nature 1989; 342: 238-243

13. Kendler KS, Karkowski LM et al. The structure of psychosis. Arch Gen Psychiatry 1998; 55: 492-499

14. Kendler KS, Pedersen $\mathrm{N}$ et al. A pilot Swedish twin study of affective illness, including hospital- and population-ascertained subsamples. Arch Gen Psychiatry 1993; 50 : 699-700
15. Maier W, Lichtermann D et al. Continuity and discontinuity of affective disorders and schizophrenia. Results of a controlled family. Arch Gen Psychiatry 1993; 50: 871-883

16. McGuffin P, Katz R et al. A hospital-based twin register of the heritability of DSMIV unipolar depression. Arch Gen Psychiatry 1996; 53: 129-136

17. Mendelbaum K, Sevy S et al. Manic-depressive illness and linkage reanalysis in the Xq27-Xq28 region of chromosome $X$. Neuropsychobiology 1995; 31: 58-63

18. Mendelwicz J, Linkowski P et al. Linkage between glucose- 6 -phosphate dehydrogenase deficiency and manic-depressive psychosis. Br J Psychiatry 1980; 137:337-342

19. Mendlewicz J und Rainer JD. Adoption study supporting genetic transmission in manic-depressive illness. Nature 1977; 268 : 327-329

20. Rice J, Reich T et al. The familial transmission of bipolar illness. Arch Gen Psychiatry 1987; 44: 441-447

21. Schuhmacher J, Cichon S et al. Genetik bipolar affektiver Störungen - Gegenwärtiger Stand der Arbeiten zur Identifikation von Dispositionsgenen. Nervenarzt 2002; 73:581-894

22. Schumacher J, Abou Jamra R et al. G72 and $\mathrm{D}$-amino acid oxidase are shared genetic risk factors for schizophrenia and bipolar affective disorder (eingereicht)

23. Schwab SG, Knapp M et al. Support for association of schizophrenia with genetic variation in the 6 p22.3 gene, dysbindin, in sib-pair families with linkage and in an additional sample of triad families. Am J Hum Genet 2003; 72: 185-190

24. Segurado R, Detera-Wadleigh SD et al. Genome scan meta-analysis of bipolar disorder. Am J Med Genet 2002; 114: 701

25. Sklar P. Linkage analysis in psychiatric disorders: the emerging picture. Annu Rev Genomics Hum Genet 2002; 3: 371-413

26. Stefansson H, Sarginson ] et al. Association of neuregulin 1 with schizophrenia confirmed in a Scottish population. Am J Hum Genet 2003; 72: 83-87

27. Wender PH, Kety SS et al. Psychiatric disorders in the biological and adoptive families of adopted individuals with affective disorders. Arch Gen Psychiatry 1986; 43 : 923-929

\section{Korrespondenzadresse:}

Prof. Dr. Marcella Rietschel

Zentralinstitut für Seelische Gesundheit J5

68159 Mannheim

rietschel@zi-mannheim.de 